\title{
Genetic Diversity among Promising Lines and their F1 Progeny of Sponge Gourd [Luffa cylindrica (Roem) L.]
}

\author{
Yamuna Prasad Singh*, V.B. Singh, Praveen Kumar Singh, \\ Vimlesh Kumar and Ram Parsad
}
Department of Vegetable Science, Narendra Deva University of Agriculture and Technology, Narendra Nagar, Kumarganj, Faizabad (U.P.) 224 229, India
*Corresponding author

\section{A B S T R A C T}

\begin{tabular}{|l|}
\hline K e y w o r d s \\
Sponge gourd, \\
Genetic \\
divergence, \\
D2 analysis, \\
Cluster analysis. \\
\hline Article Info \\
\hline $\begin{array}{l}\text { Accepted: } \\
\text { 29 June } 2017 \\
\text { Available Online: } \\
\text { 10 July } 2017\end{array}$ \\
\hline
\end{tabular}

\section{Introduction}

Luffa (Luffa cylindrica (L.) MJ. Roem syn. L. aegyptica Mill.) are domesticated species and commonly called as sponge gourd, loofah, vegetable sponge or dish cloth. It belongs to
The investigation revealed that the 54 genotypes $\left(10\right.$ lines +4 testers $+40 \mathrm{~F}_{1}$ 's $)$ of sponge gourd in randomized block design with three replications during two seasons were grouped into 8 distinct non over lapping clusters which indicated presence of considerable diversity among the genotypes during both seasons, which shows clusters contained genotypes of heterogenous origin. It is also suggested that there is no parallelism between genetic and geographic diversity. Cluster I had highest number of genotypes (32 and 36) while, the monogenotypic clusters II, III, IV and VI (1 and 1) having minimum genotype in both the years, respectively. In $\mathrm{Y}_{1}$, the intra-cluster $\mathrm{D}^{2}$ values ranged from 0.00 to 420.45 while the inter-cluster values ranged from 63.09 to 504.73. The highest intra-cluster distance was 420.45 for cluster VIII, while lowest one was zero for monogenotypic clusters II, III, IV and VI. The maximum inter-cluster distance was observed between cluster II and cluster VIII (504.73). In $\mathrm{Y}_{2}$, the intra-cluster $\mathrm{D}^{2}$ values ranged from 0.00 to 151.57 while the intercluster value varied from 57.56 to 426.59 . The highest intra-cluster distance was shown by cluster VIII (151.57), whereas it was zero for mono-genotype clusters II, III IV and VI. The maximum inter-cluster distance was observed between cluster V and cluster VIII (426.59) suggesting that the genotypes/ $\mathrm{F}_{1}$ 's belonging to these clusters may be used as parents/crosses for hybridization programme to develop desirable type $F_{1}$ or segregates because crosses between genetically divergent lines/crosses will generate heterotic $\mathrm{F}_{1}{ }^{\mathrm{s}}$. Highest inter-cluster value indicated that the selected breeding lines were highly divergent. Cluster means for different traits indicated considerable differences between the clusters. Cluster VI, VII and VIII in $\mathrm{Y}_{1}$ and cluster V, II and III in $\mathrm{Y}_{2}$ had in general medium mean performance for most of the characters. Maximum cluster means for average fruit yield per plant was observed in cluster II followed by cluster VI in $\mathrm{Y}_{1}$, while in $\mathrm{Y}_{2}$ cluster VI recorded maximum cluster means for average fruit yield followed by cluster I. In $\mathrm{Y}_{1}$, vine length $(\mathrm{m})$ contributed maximum contribution towards total divergence while minimum contribution was reflected by days to first fruit harvest in both the years, respectively. 
rainy and summer season vegetable which is grown throughout the country and world. It originated in subtropical Asian region particularly India (Kaloo, 1993). Sponge gourd is an annual and monoeceous cucurbit plant and it has a gelatinous compound luffien. The nutritive value of sponge gourd fruits per $100 \mathrm{~g}$ edible portion (tough skin removed, edible portion $80 \%$ ) is: water 93.2 $\mathrm{g}$, energy $18 \mathrm{kcal}$, protein $1.2 \mathrm{~g}$, fat $0.2 \mathrm{~g}$, carbohydrate $2.9 \mathrm{~g}$, fibre $2.0 \mathrm{~g}$, Ca $36 \mathrm{mg}, \mathrm{P}$ $19 \mathrm{mg}, \mathrm{Fe} 1.1 \mathrm{mg}$, carotene $120 \mu \mathrm{g}$, thiamine $0.02 \mathrm{mg}$, riboflavin $0.06 \mathrm{mg}$, niacin $0.4 \mathrm{mg}$ and the composition of young leaves per 100 g edible portion is: water $89 \mathrm{~g}$, protein $5.1 \mathrm{~g}$, carbohydrate $4.0 \mathrm{~g}$, fibre $1.5 \mathrm{~g}$, Ca $56 \mathrm{mg}, \mathrm{Fe}$ $11.5 \mathrm{mg}$, carotene $9.2 \mathrm{mg}$, ascorbic acid 95 mg. It used for scrubbing of body skin as a bath sponge increased blood circulation and utensils purposes. The tender fruits are rich in vitamin $\mathrm{A}$, vitamin $\mathrm{C}$ and iron (Yawalkar, 2004). It has certain medicinal uses. The cooked fruits are easily digestible and very appetizing, therefore, it is recommended to the patients suffering from malaria or other seasonal fevers. Among vegetables, cucurbits are associated with the origin of agriculture and dawn of human civilization. In food crops, cucurbits are largest producer of biological water and easily digestive and recommended even to sick and frail patients. Its flowers are yellow in colour and showy having five petals. The inflorescences of staminate flowers are raceme, while pistillate flowers are solitary and short long pendunculate. It produces fruits containing a fibrous vascular system having vigorous vines with cylindrical ten angled fruits, (Whitaker and Davis, 1962). To develop a new variety there is need of high magnitude of genetic variability in the base material and the vast of variability for desired characters. A good knowledge on genetic diversity or genetic similarity could be helpful in long term selection gain in plants (Kumar et al., 2012). Hence, genetic variability and diversity is of prime interest to the plant breeder as it plays a key role in framing a successful breeding programme. The genetically diverse parents are always able to produce high heterotic effects and great frequency of desirable segregants in further generations as already reported by Kumar et al., (1994). D ${ }^{2}$ statistic is a useful tool to measure genetic divergence among genotypes in any crop as developed by Mahalanobis (1936). However, in the present study, an attempt has been made to identify genetically divergent promising lines and their $F_{1}$ progenies, so as to select the potential parents for breeding programme to attain the anticipated improvement in fruit yield per plant of sponge gourd either by crossing two dissimilar parents to get heterotic $F_{1}$ or by making single cross, three way cross, double cross and selfing of crosses $\left(\mathrm{F}_{1}\right)$ obtained by the divergent parents of sponge gourd.

\section{Materials and Methods}

The experimental materials consisted of 14 promising parental lines of sponge gourd and their $F_{1}$ progenies. Out of these advanced breeding parental lines 10 parents were choosen as lines and four as testers and crossed as per $\mathrm{L} \times \mathrm{T}$ design to get $40 \mathrm{~F}_{1}$ 's. These experimental materials were grown under Randomized Block Design (RBD) with three replications at Main Experiment Station, Department of Vegetable Science, Narendra Deva University of Agriculture and Technology, Narendra Nagar, Kumarganj, Faizabad (U.P.) India. The treatments were sown in rows spaced 2.50 meters apart with a plant to plant spacing of 0.50 meter during

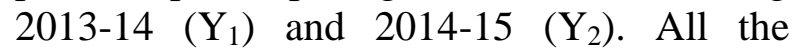
recommended agronomic package of practices and protection measures were followed to raise a good crop. Fertilizers and manures were applied as per recommended dose. Observations were recorded on all the six plants maintained carefully in each plot for fourteen quantitative characters viz., node 
number to anthesis of first staminate flower, node number to anthesis of first pistillate flower, days to anthesis of first staminate flower, days to anthesis of first pistillate flower, node number of first fruit harvest, days to first fruit harvest, number of primary branches per plant, inter nodal length $(\mathrm{cm})$, vine length $(\mathrm{m})$, fruit length $(\mathrm{cm})$, fruit circumference $(\mathrm{cm})$, average fruit weight $(\mathrm{g})$, number of fruits per plant and average fruits yield per plant $(\mathrm{kg})$. Analysis of variance was carried out as suggested by Panse and Sukhatme (1967). Clustering of genotypes was done according to Tocher's method as described by Rao (1952). The per cent contribution of characters towards genetic divergence was calculated according to Singh and Chaudhary (1985). Genetic divergence was estimated by using $\mathrm{D}^{2}$ statistics of Mahalanobis (1936).

\section{Results and Discussion}

The analysis of variance (Table 1) revealed that the significant differences were present for all the characters studied and the experimental materials were genetically divergent from each other both $\left(\mathrm{Y}_{1}\right.$ and $\left.\mathrm{Y}_{2}\right)$ the years which indicated that there is ample scope for selection of promising lines and their $F_{1}$ progenies from the present gene pool aimed at enhancing genetic yield potential of sponge gourd. All the fifty four genotypes were grouped into 8 different non over lapping clusters following Mahalanobis's methods (Table 2). Cluster I had highest number of genotypes (32 and 36) while, the monogenotypic clusters II, III, IV and VI (1 and 1) having minimum genotype in both the years, respectively. The estimates of intra and inter-cluster distances represented by $\mathrm{D}^{2}$ values are given in table 3 . In $\mathrm{Y}_{1}$, the intracluster $\mathrm{D}^{2}$ values ranged from 0.00 to 420.45 while the inter-cluster values ranged from 63.09 to 504.73 . The highest intra-cluster distance was 420.45 for cluster VIII, while lowest one was zero for monogenotypic clusters II, III, IV and VI. The maximum inter-cluster distance was observed between cluster II and cluster VIII (504.73). In $\mathrm{Y}_{2}$, the intra-cluster $\mathrm{D}^{2}$ values ranged from 0.00 to 151.57 while the inter-cluster value varied from 57.56 to 426.59 . The highest intracluster distance was shown by cluster VIII (151.57), whereas it was zero for monogenotype clusters II, III IV and VI. The maximum inter-cluster distance was observed between cluster V and cluster VIII (426.59) suggesting that the genotypes $/ \mathrm{F}_{1}$ 's belonging to these clusters may be used as parents/crosses for hybridization programme to develop desirable type $F_{1}$ or segregates because crosses between genetically divergent lines/crosses will generate heterotic $\mathrm{F}_{1}{ }^{\mathrm{s}}$ Varalakshmi et al., (1994), Mathew et al., (2001), Karuppaiah et al., (2005), Islam et al., (2010) and Resmi et al., (2012) also reported similar findings. As heterosis can be best exploited and chances of getting transgressive segregants are maximum when generating diverse lines are crossed. High inter-cluster value indicated that the selected breeding lines were highly divergent in both the years (Sundaram and Vadivel (2007) and Singh et al., (2014).

Highest inter-cluster value indicated that the selected breeding lines were highly divergent. The comparison of clusters means revealed considerable differences among the clusters of different quantitative characters (Table 4). Cluster VIII showed high mean value for maximum five characters viz., node number to anthesis of first staminate flower (10.47), node number to anthesis of first pistillate flower (14.13), days to anthesis of first staminate flower (41.20), node number of first fruit harvest (14.46) and days to first fruit harvest (50.97), cluster II showed high mean value for fruit circumference (9.30), number of fruits per plant (37.63) and average fruits yield per plant (3.87) and cluster VII showed high mean value for inter nodal length (7.71) and average fruit weight (172.65). 
Table.1 Analysis of variance for yield and its component traits in sponge gourd (Y1=2013-14 and Y2=2014-15)

\begin{tabular}{|c|c|c|c|c|c|c|c|c|}
\hline \multirow{4}{*}{$\begin{array}{l}\text { S. } \\
\text { No. }\end{array}$} & \multirow{4}{*}{ Characters } & \multicolumn{7}{|c|}{ Mean sum of Squares } \\
\hline & & \multirow{2}{*}{$\begin{array}{c}\begin{array}{c}\text { Source of } \\
\text { variation }\end{array} \\
\text { Years }\end{array}$} & \multicolumn{2}{|c|}{ Replications } & \multicolumn{2}{|c|}{ Treatments } & \multicolumn{2}{|c|}{ Error } \\
\hline & & & $\mathbf{Y}_{1}$ & $\mathbf{Y}_{2}$ & $\mathbf{Y}_{1}$ & $\mathbf{Y}_{2}$ & $\mathbf{Y}_{1}$ & $\mathbf{Y}_{2}$ \\
\hline & & $d f$ & 2 & 2 & 53 & 53 & 106 & 106 \\
\hline 1. & $\begin{array}{l}\text { Node no.to anthesis of first staminate } \\
\text { flower }\end{array}$ & & 0.07 & 0.10 & $12.09 * *$ & $15.39 * *$ & 0.13 & 0.12 \\
\hline 2. & $\begin{array}{l}\text { Node no.to anthesis of first pistillate } \\
\text { flower }\end{array}$ & & 0.39 & 0.56 & $18.17 * *$ & $18.06^{* *}$ & 0.32 & 0.31 \\
\hline 3. & Days to anthesis of first staminate flower & & 0.14 & 3.38 & $85.67 * *$ & $84.87 * *$ & 3.73 & 3.53 \\
\hline 4. & Days to anthesis of first pistillate flower & & 2.10 & 1.38 & $75.00 * *$ & $70.83 * *$ & 4.02 & 4.16 \\
\hline 5. & Node no. of first fruit harvest & & 0.67 & 0.35 & $18.38 * *$ & $16.55^{* *}$ & 0.32 & 0.32 \\
\hline 6. & Days to first fruit harvest & & 7.47 & 8.75 & $66.32 * *$ & $66.37 * *$ & 7.32 & 7.08 \\
\hline 7. & No.of primary branches per plant & & 0.09 & 0.16 & $3.92 * *$ & $4.51 * *$ & 0.15 & 0.18 \\
\hline 8. & Inter nodal length $(\mathrm{cm})$ & & 0.41 & 0.00 & $5.79 * *$ & $5.59 * *$ & 0.20 & 0.19 \\
\hline 9. & Vine length (m) & & 0.02 & 0.07 & $3.78 * *$ & $3.27 * *$ & 0.06 & 0.06 \\
\hline 10. & Fruit length $(\mathrm{cm})$ & & 0.57 & 0.08 & $47.29 * *$ & $43.56^{* *}$ & 2.24 & 2.26 \\
\hline 11. & Fruit circumference $(\mathrm{cm})$ & & 34.00 & 0.36 & $1.16^{* *}$ & $1.48 * *$ & 0.30 & 0.31 \\
\hline 12. & Average fruit weight (g) & & 132.48 & 23.15 & $1361.00 * *$ & $1131.41 * *$ & 60.43 & 57.29 \\
\hline 13. & No. of fruits per plant & & 6.65 & 2.11 & $93.07 * *$ & $93.10 * *$ & 2.60 & 2.43 \\
\hline 14. & Average fruits yield per plant (kg) & & 0.06 & 0.02 & $0.96 * *$ & $1.16^{* *}$ & 0.05 & 0.04 \\
\hline
\end{tabular}


Table.2 Clustering pattern of fifty four genotypes of sponge gourd on the basis of Mahalnobis 'D2' statistics (Y1=2013-14 and Y2=2014-15)

\begin{tabular}{|c|c|c|c|}
\hline $\begin{array}{l}\text { Cluster } \\
\text { number }\end{array}$ & Years & $\begin{array}{l}\text { No. of } \\
\text { genotypes }\end{array}$ & Genotypes \\
\hline \multirow[b]{2}{*}{ I } & $\mathrm{Y}_{1}$ & 32 & $\begin{array}{l}\text { NDSG-21 x NDSG-11, NDSG-55 x NDSG-15, NDSG-21 x NDSG-15, NDSG-4 x NDSG-15, NDSG-6 x NDSG-15, } \\
\text { NDSG-63 x NDSG-15, NDSG-2 x NDSG-15, NDSG-6 x Pusa Chikni(c), NDSG-21 x Pusa Chikni(c), NDSG-63 x } \\
\text { NDSG-12, NDSG-2 x NDSG-11, NDSG-6 x NDSG-12, NDSG-1 x NDSG-11, NDSG-55 x Pusa Chikni(c), NDSG-6 } \\
\text { x NDSG-11, NDSG-1 x Pusa Chikni(c), NDSG-24 x NDSG-11, NDSG-55, NDSG-55 X NDSG-12, NDSG-18 X } \\
\text { NDSG-15, NDSG-1 X NDSG-15, NDSG-63 X Pusa Chikni, NDSG-24 X NDSG-12, NDSG-1 X NDSG-12, NDSG-2 } \\
\text { X NDSG-12, NDSG-12, NDSG-18 X NDSG-12, Pusa Chikni C, NDSG-55 X NDSG-11, NDSG-10 X Pusa Chikni, } \\
\text { NDSG-11, NDSG-4 X NDSG-11 }\end{array}$ \\
\hline & $\mathrm{Y}_{2}$ & 36 & $\begin{array}{l}\text { NDSG-21 X NDSG-15, NDSG-55 X NDSG-15, NDSG-4 X NDSG-15, NDSG-2 X NDSG-15, NDSG-6 X NDSG-15, } \\
\text { NDSG-21 X NDSG-11, NDSG-63 X NDSG-15, NDSG-63 X NDSG-12, NDSG-2 X NDSG-11, NDSG-1 X NDSG- } \\
11 \text {, NDSG-18 X NDSG-15, NDSG-55 X Pusa Chikni, NDSG-6 X NDSG-12, NDSG-2 X NDSG-12, NDSG-1 X } \\
\text { NDSG-12, NDSG-21 X Pusa Chikni, NDSG-1 X NDSG-15, NDSG-55 X NDSG-12, NDSG-55 X NDSG-11, NDSG- } \\
1 \text { X Pusa Chikni, NDSG-6 X Pusa Chikni, NDSG-6 X NDSG-11, NDSG-10 X Pusa Chikni, NDSG-24 X NDSG-12, } \\
\text { NDSG-10 X NDSG-12, NDSG-63 X Pusa Chikni, Pusa Chikni @ C, NDSG-12, NDSG-55, NDSG-18 X NDSG-12, } \\
\text { NDSG-1, NDSG-24 X NDSG-11, NDSG-21 X NDSG-12, NDSG-10 X NDSG-15, NDSG-4 X NDSG-11, NDSG-24 } \\
\text { X NDSG-15 }\end{array}$ \\
\hline \multirow{2}{*}{ II } & $\mathrm{Y}_{1}$ & 1 & NDSG-18 X NDSG-11 \\
\hline & $\mathrm{Y}_{2}$ & 1 & NDSG-11 \\
\hline \multirow{2}{*}{ III } & $\mathrm{Y}_{1}$ & 1 & NDSG-21 X NDSG-12 \\
\hline & $\mathrm{Y}_{2}$ & 1 & NDSG-2 X Pusa Chikni \\
\hline \multirow{2}{*}{ IV } & $\mathrm{Y}_{1}$ & 1 & NDSG-24 X NDSG-15 \\
\hline & $\mathrm{Y}_{2}$ & 1 & NDSG-4 X Pusa Chikni \\
\hline \multirow[t]{2}{*}{$\mathbf{V}$} & $\mathrm{Y}_{1}$ & 9 & $\begin{array}{l}\text { NDSG-4 X NDSG-12, NDSG-63 X NDSG-11, NDSG-4 X Pusa Chikni, NDSG-24 X Pusa Chikni, NDSG-18 X Pusa } \\
\text { Chikni, NDSG-10 X NDSG-12, NDSG-18, NDSG-24, NDSG-4 }\end{array}$ \\
\hline & $\mathrm{Y}_{2}$ & 3 & NDSG-10 X NDSG-11, NDSG-10, NDSG-4 \\
\hline \multirow{2}{*}{ VI } & $\mathrm{Y}_{1}$ & 1 & NDSG-1 \\
\hline & $\mathrm{Y}_{2}$ & 1 & NDSG-24 X Pusa Chikni \\
\hline \multirow[b]{2}{*}{ VII } & $\mathrm{Y}_{1}$ & 4 & NDSG-10 X NDSG-15, NDSG-10, NDSG-10 X NDSG-11, NDSG-2 X Pusa Chikni \\
\hline & $\mathrm{Y}_{2}$ & 6 & $\begin{array}{l}\text { NDSG-4 X NDSG-12, NDSG-63 X NDSG-11, NDSG-18, NDSG-18 X Pusa Chikni, NDSG-24, NDSG-18 X NDSG- } \\
11\end{array}$ \\
\hline \multirow{2}{*}{ VIII } & $\mathrm{Y}_{1}$ & 5 & NDSG-6, NDSG-63, NDSG-2, NDSG-21, NDSG-15 \\
\hline & $\mathrm{Y}_{2}$ & 5 & NDSG-6, NDSG-63, NDSG-2, NDSG-21, NDSG-15 \\
\hline
\end{tabular}


Table.3 Intra-inter clusters D2 values for eight clusters in sponge gourd (Y1=2013-14 and Y2=2014-15)

\begin{tabular}{|c|c|c|c|c|c|c|c|c|c|}
\hline & Years & Cluster I & Cluster II & Cluster III & Cluster IV & Cluster V & Cluster VI & Cluster VII & $\begin{array}{c}\text { Cluster } \\
\text { VIII }\end{array}$ \\
\hline \multirow{2}{*}{ Cluster I } & $\mathrm{Y}_{1}$ & 45.37 & 75.33 & 83.19 & 68.15 & 95.73 & 79.98 & 102.49 & 350.25 \\
\hline & $\mathrm{Y}_{2}$ & 52.12 & 90.43 & 75.22 & 75.40 & 108.99 & 88.90 & 107.36 & 324.47 \\
\hline \multirow{2}{*}{ Cluster II } & $\mathrm{Y}_{1}$ & & 0.00 & 182.34 & 117.52 & 139.38 & 162.52 & 170.76 & 504.73 \\
\hline & $\mathrm{Y}_{2}$ & & 0.00 & 69.14 & 87.57 & 73.71 & 190.84 & 121.15 & 417.83 \\
\hline \multirow{2}{*}{ Cluster III } & $\mathrm{Y}_{1}$ & & & 0.00 & 104.09 & 132.17 & 72.10 & 133.15 & 226.84 \\
\hline & $\mathrm{Y}_{2}$ & & & 0.00 & 57.56 & 74.60 & 115.31 & 114.66 & 258.20 \\
\hline \multirow{2}{*}{ Cluster IV } & $\mathrm{Y}_{1}$ & & & & 0.00 & 63.09 & 150.19 & 112.92 & 376.85 \\
\hline & $\mathrm{Y}_{2}$ & & & & 0.00 & 90.69 & 77.84 & 87.34 & 381.39 \\
\hline \multirow{2}{*}{ Cluster V } & $\mathrm{Y}_{1}$ & & & & & 56.79 & 156.65 & 155.59 & 422.21 \\
\hline & $\mathrm{Y}_{2}$ & & & & & 45.13 & 192.84 & 171.88 & 426.59 \\
\hline \multirow{2}{*}{ Cluster VI } & $\mathrm{Y}_{1}$ & & & & & & 0.00 & 167.28 & 226.01 \\
\hline & $\mathrm{Y}_{2}$ & & & & & & 0.00 & 100.37 & 343.85 \\
\hline \multirow{2}{*}{ Cluster VII } & $\mathrm{Y}_{1}$ & & & & & & & 100.38 & 420.45 \\
\hline & $\mathrm{Y}_{2}$ & & & & & & & 98.80 & 392.30 \\
\hline \multirow{2}{*}{ Cluster VIII } & $\mathrm{Y}_{1}$ & & & & & & & & 420.45 \\
\hline & $\mathrm{Y}_{2}$ & & & & & & & & 151.57 \\
\hline
\end{tabular}


Table.4 Intra-cluster group mean for 14 clusters in sponge gourd (Y1=2013-14 and Y2=2014-15)

\begin{tabular}{|c|c|c|c|c|c|c|c|c|}
\hline Cluster number & Years & $\begin{array}{l}\text { Node no.to } \\
\text { anthesis of } \\
\text { first staminate } \\
\text { flower }\end{array}$ & $\begin{array}{l}\text { Node no.to } \\
\text { anthesis of } \\
\text { first pistillate } \\
\text { flower }\end{array}$ & $\begin{array}{l}\text { Days to } \\
\text { anthesis of } \\
\text { first staminate } \\
\text { flower }\end{array}$ & $\begin{array}{l}\text { Days to } \\
\text { anthesis of first } \\
\text { pistillate flower }\end{array}$ & $\begin{array}{l}\text { Node no. of } \\
\text { first fruit } \\
\text { harvest }\end{array}$ & $\begin{array}{l}\text { Days to first } \\
\text { fruit harvest }\end{array}$ & $\begin{array}{l}\text { No.of primary } \\
\text { branches per } \\
\text { plant }\end{array}$ \\
\hline \multirow{2}{*}{ I } & $\mathrm{Y}_{1}$ & 4.59 & 7.59 & 30.23 & 34.28 & 8.29 & 45.98 & 6.31 \\
\hline & $\mathrm{Y}_{2}$ & 4.53 & 7.77 & 30.74 & 34.94 & 8.34 & 46.20 & 6.36 \\
\hline \multirow{2}{*}{ II } & $Y_{1}$ & 3.87 & 6.10 & 21.17 & 25.80 & 6.13 & 38.17 & 7.50 \\
\hline & $\mathrm{Y}_{2}$ & 3.83 & 8.17 & 20.70 & 29.53 & 8.07 & 42.50 & 8.13 \\
\hline \multirow{2}{*}{ III } & $Y_{1}$ & 4.50 & 12.20 & 30.07 & 38.17 & 13.30 & 50.33 & 4.87 \\
\hline & $\mathrm{Y}_{2}$ & 4.53 & 10.13 & 34.13 & 37.27 & 10.63 & 51.27 & 6.50 \\
\hline \multirow{2}{*}{ IV } & $\mathrm{Y}_{1}$ & 4.80 & 7.33 & 31.47 & 32.33 & 8.03 & 44.27 & 7.17 \\
\hline & $\mathrm{Y}_{2}$ & 4.23 & 7.10 & 25.37 & 31.67 & 8.00 & 54.63 & 7.20 \\
\hline \multirow{2}{*}{$\mathbf{V}$} & $\mathrm{Y}_{1}$ & 4.24 & 7.63 & 27.60 & 32.48 & 8.57 & 45.71 & 6.79 \\
\hline & $Y_{2}$ & 3.44 & 7.21 & 26.03 & 29.76 & 7.97 & 43.61 & 6.01 \\
\hline \multirow{2}{*}{ VI } & $\mathrm{Y}_{1}$ & 6.67 & 8.33 & 36.47 & 41.70 & 10.07 & 51.70 & 4.07 \\
\hline & $Y_{2}$ & 4.23 & 8.23 & 31.27 & 37.13 & 8.93 & 50.27 & 7.70 \\
\hline \multirow{2}{*}{ VII } & $\mathrm{Y}_{1}$ & 3.68 & 7.99 & 28.13 & 30.85 & 8.77 & 42.55 & 6.03 \\
\hline & $\mathrm{Y}_{2}$ & 4.30 & 7.77 & 27.52 & 30.39 & 8.74 & 44.24 & 7.38 \\
\hline \multirow{2}{*}{ VIII } & $\mathrm{Y}_{1}$ & 10.47 & 14.13 & 41.20 & 41.36 & 14.46 & 50.97 & 5.89 \\
\hline & $\mathrm{Y}_{2}$ & 11.62 & 14.29 & 40.37 & 40.19 & 14.67 & 50.30 & 5.89 \\
\hline
\end{tabular}

\begin{tabular}{|c|c|c|c|c|c|c|c|c|}
\hline Cluster number & Years & $\begin{array}{l}\text { Inter nodal } \\
\text { length }(\mathrm{cm})\end{array}$ & $\begin{array}{l}\text { Vine length } \\
\text { (m) }\end{array}$ & $\begin{array}{l}\text { Fruit length } \\
\text { (cm) }\end{array}$ & $\begin{array}{l}\text { Fruit } \\
\text { circumference } \\
\text { (cm) }\end{array}$ & $\begin{array}{l}\text { Average fruit } \\
\text { weight (g) }\end{array}$ & $\begin{array}{l}\text { No. of fruits } \\
\text { per plant }\end{array}$ & $\begin{array}{l}\text { Average fruits } \\
\text { yield per plant } \\
\text { (kg) }\end{array}$ \\
\hline \multirow{2}{*}{ I } & $\mathrm{Y}_{1}$ & 6.94 & 2.95 & 24.07 & 8.79 & 143.47 & 26.19 & 3.21 \\
\hline & $\mathrm{Y}_{2}$ & 7.13 & 3.04 & 23.99 & 8.80 & 146.80 & 25.36 & 3.18 \\
\hline \multirow{2}{*}{ II } & $\mathrm{Y}_{1}$ & 6.40 & 2.50 & 23.33 & 9.30 & 125.07 & 37.63 & 3.87 \\
\hline & $\mathrm{Y}_{2}$ & 6.20 & 2.37 & 22.23 & 7.80 & 115.07 & 24.63 & 2.46 \\
\hline \multirow{2}{*}{ III } & $\mathrm{Y}_{1}$ & 7.60 & 3.63 & 21.03 & 9.27 & 135.73 & 27.27 & 3.16 \\
\hline & $Y_{2}$ & 4.37 & 3.33 & 23.37 & 8.23 & 145.10 & 16.47 & 2.08 \\
\hline \multirow{2}{*}{ IV } & $\mathrm{Y}_{1}$ & 8.30 & 5.33 & 18.43 & 8.37 & 130.27 & 22.23 & 2.56 \\
\hline & $\mathrm{Y}_{2}$ & 4.93 & 4.40 & 27.20 & 7.60 & 170.27 & 17.47 & 2.79 \\
\hline \multirow{2}{*}{$\mathbf{V}$} & $\mathrm{Y}_{1}$ & 6.51 & 5.13 & 23.85 & 8.85 & 148.84 & 22.76 & 2.87 \\
\hline & $\mathrm{Y}_{2}$ & 7.56 & 2.54 & 20.04 & 8.27 & 159.90 & 11.99 & 1.73 \\
\hline \multirow{2}{*}{ VI } & $\mathrm{Y}_{1}$ & 4.50 & 2.10 & 26.40 & 7.87 & 138.43 & 26.53 & 3.28 \\
\hline & $\mathrm{Y}_{2}$ & 7.60 & 5.93 & 20.43 & 8.33 & 170.50 & 25.33 & 4.12 \\
\hline \multirow{2}{*}{ VII } & $\mathrm{Y}_{1}$ & 7.71 & 2.63 & 19.83 & 8.33 & 172.65 & 15.20 & 2.17 \\
\hline & $\mathrm{Y}_{2}$ & 6.34 & 5.08 & 24.88 & 9.11 & 124.48 & 28.97 & 3.09 \\
\hline \multirow{2}{*}{ VIII } & $\mathrm{Y}_{1}$ & 6.40 & 3.19 & 25.80 & 8.79 & 143.98 & 22.87 & 2.83 \\
\hline & $\mathrm{Y}_{2}$ & 6.17 & 3.15 & 25.47 & 9.03 & 144.01 & 21.61 & 2.87 \\
\hline
\end{tabular}


Table.5 Per cent contribution of 14 characters towards total genetic divergence in sponge gourd $(\mathrm{Y} 1=2013-14$ and $\mathrm{Y} 2=2014-15)$

\begin{tabular}{|c|l|c|c|}
\hline \multirow{2}{*}{ S. No. } & \multicolumn{1}{|c|}{ Characters } & \multicolumn{2}{|c|}{ Contribution (\%) } \\
\cline { 3 - 4 } & & \multicolumn{1}{|c|}{$\mathbf{Y}_{\mathbf{1}}$} & $\mathbf{Y}_{\mathbf{2}}$ \\
\hline $\mathbf{1 .}$ & Node no.to anthesis of first staminate flower & 11.18 & 16.14 \\
\hline $\mathbf{2 .}$ & Node no.to anthesis of first pistillate flower & 9.64 & 4.75 \\
\hline $\mathbf{3 .}$ & Days to anthesis of first staminate flower & 0.84 & 3.35 \\
\hline $\mathbf{4 .}$ & Days to anthesis of first pistillate flower & 1.96 & 1.54 \\
\hline $\mathbf{5 .}$ & Node no. of first fruit harvest & 4.40 & 0.91 \\
\hline $\mathbf{6 .}$ & Days to first fruit harvest & 0.21 & 0.14 \\
\hline $\mathbf{7 .}$ & Inter nodal length (cm) & 12.37 & 10.69 \\
\hline $\mathbf{8 .}$ & No.of primary branches per plant & 10.13 & 7.06 \\
\hline $\mathbf{9 .}$ & Vine length (m) & 22.99 & 18.66 \\
\hline $\mathbf{1 0 .}$ & Fruit length (cm) & 5.52 & 8.46 \\
\hline $\mathbf{1 1 .}$ & Fruit circumference (cm) & 0.21 & 0.63 \\
\hline $\mathbf{1 2 .}$ & Average fruit weight (g) & 3.63 & 2.31 \\
\hline $\mathbf{1 3 .}$ & No. of fruits per plant & 14.40 & 12.37 \\
\hline $\mathbf{1 4 .}$ & Average fruits yield per plant (kg) & 2.52 & 13.00 \\
\hline
\end{tabular}

Fig.1 Per cent contribution of 14 characters towards total genetic divergence in sponge gourd (Y1=2013-14)

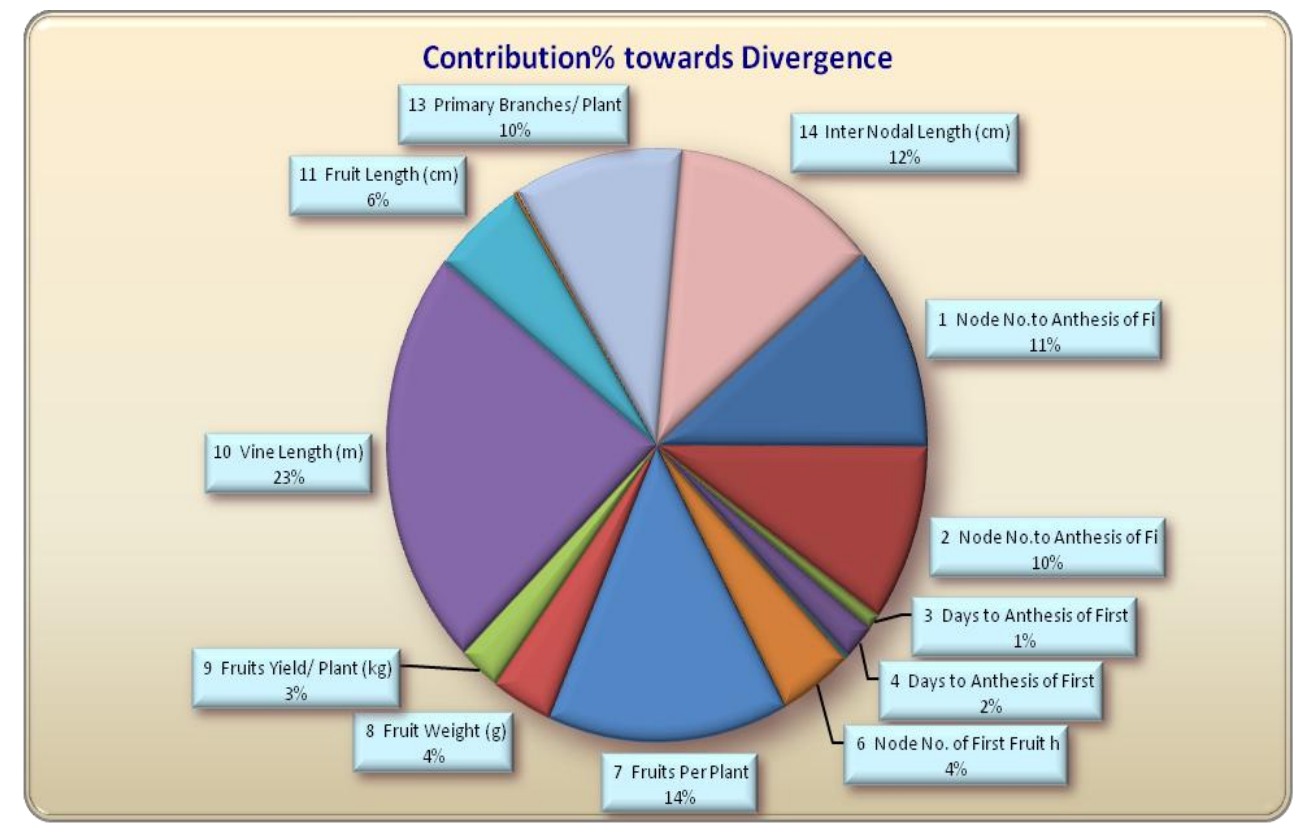


Fig.2 Per cent contribution of 14 characters towards total genetic divergence in sponge gourd $(\mathrm{Y} 2=2014-15)$

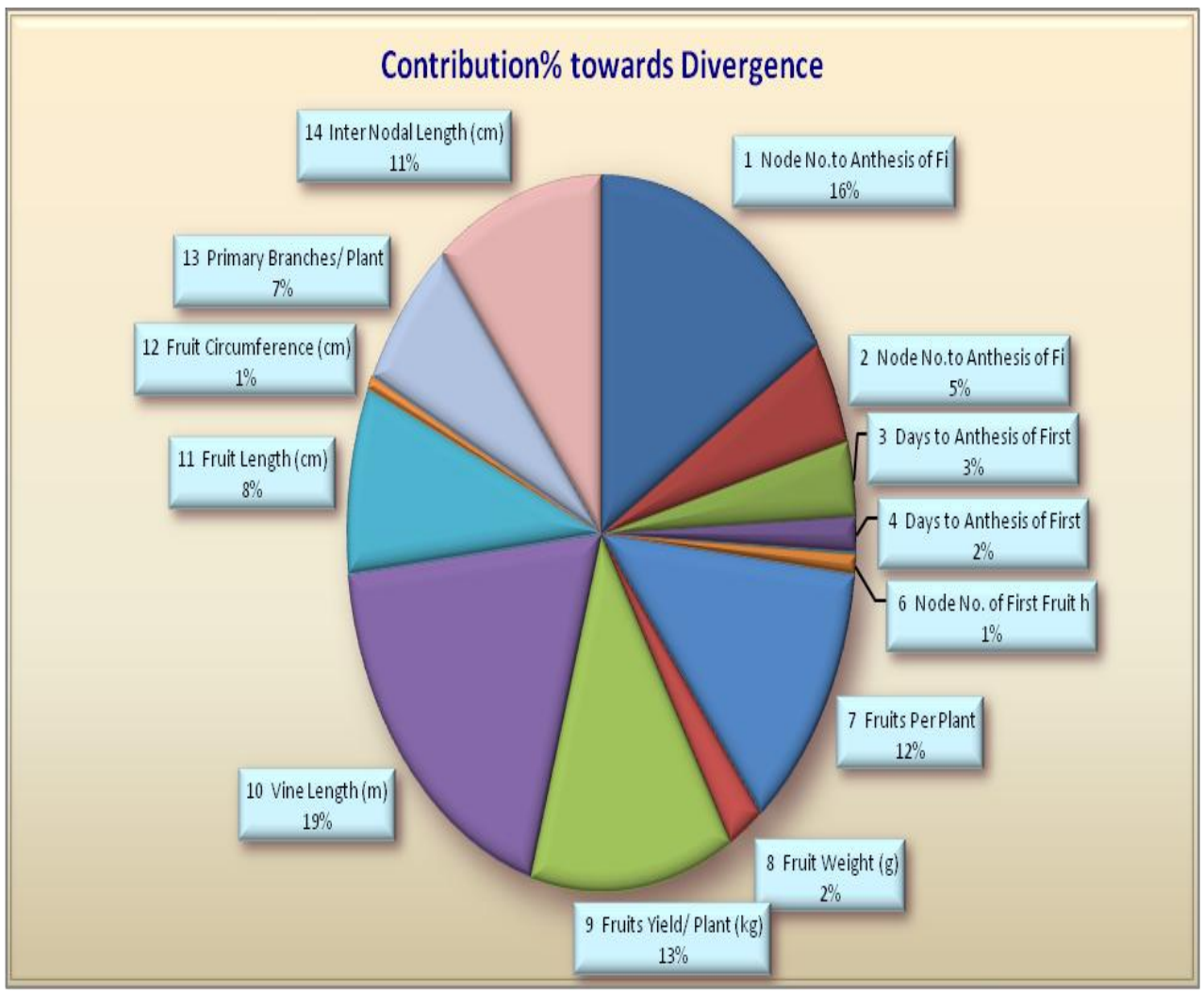

Fig.3 Intra and inter cluster distance diagram for different quantitative characters in sponge gourd by Tocher's method (Y1=2013-14)

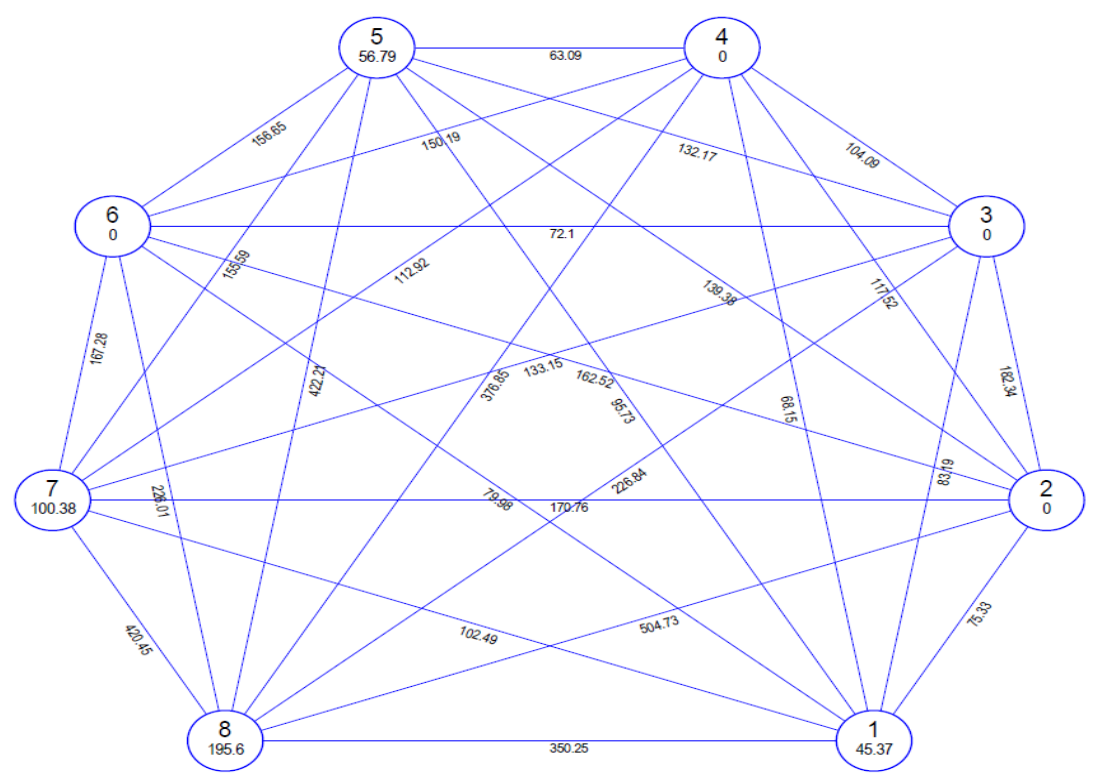

Mahalnobis Euclidean Disatnce (Not to the Scale) 
Fig.4 Clustering by Tocher's method in sponge gourd (Y1=2013-14)

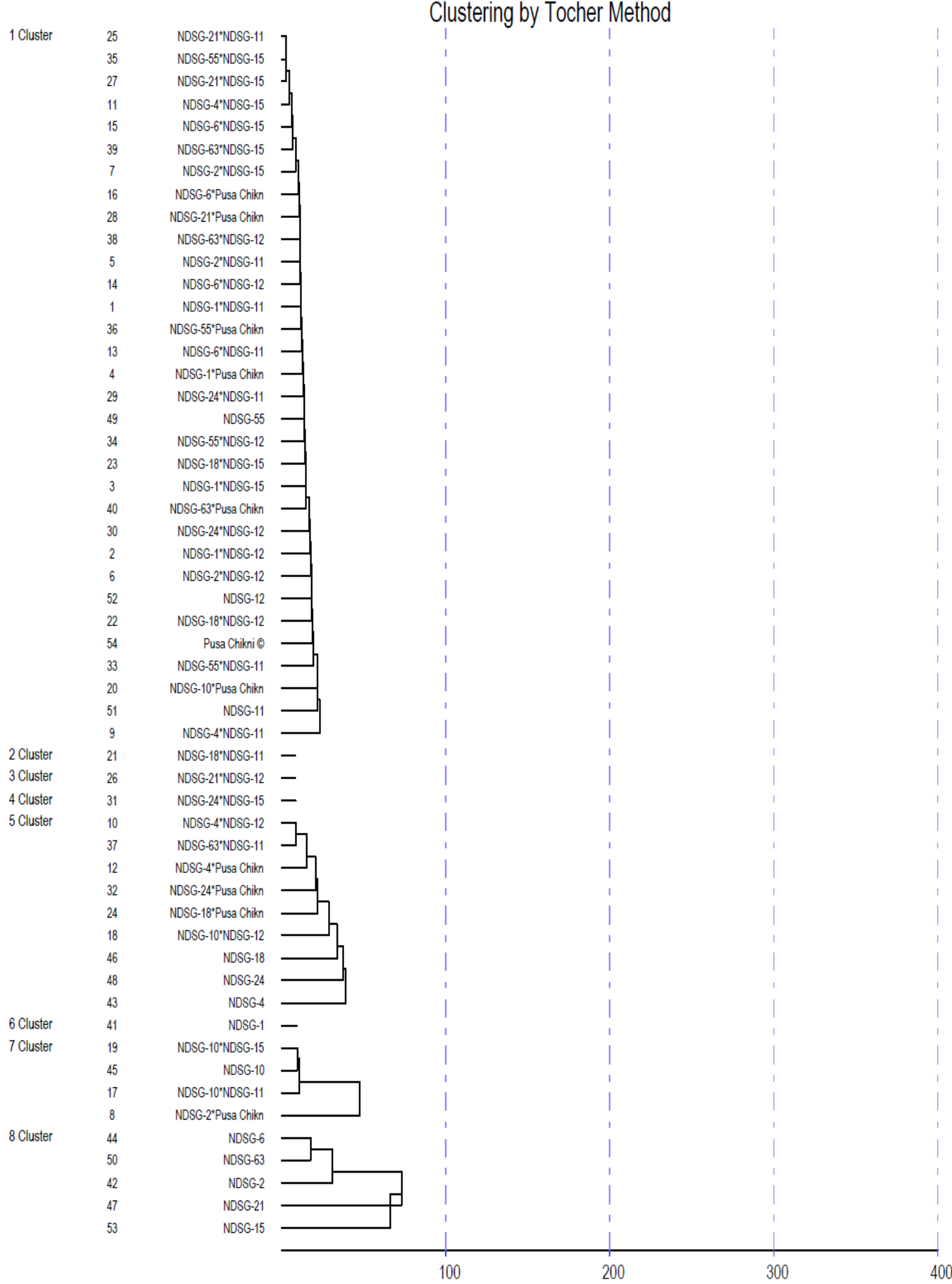


Fig.5 Clustering by Tocher's method in sponge gourd (Y2=2014-15)

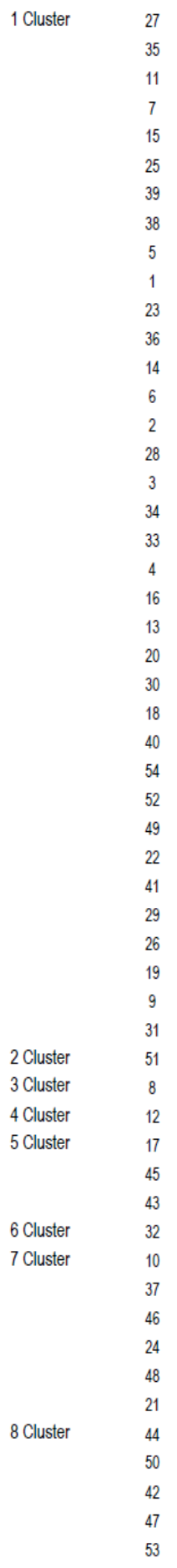

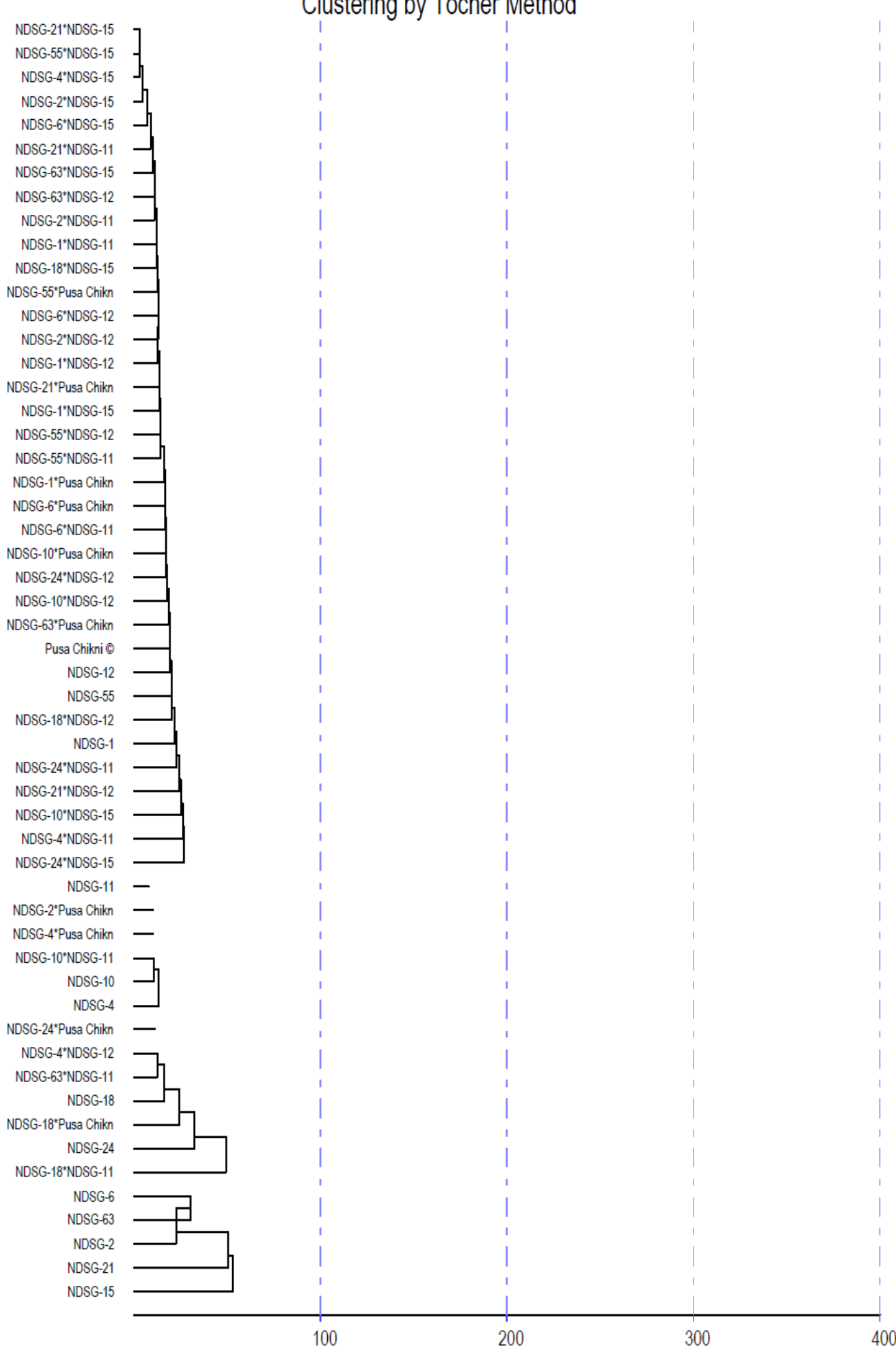


Fig.6 Intra and inter cluster distance diagram for different quantitative characters in sponge gourd by Tocher's method (Y2=2014-15)

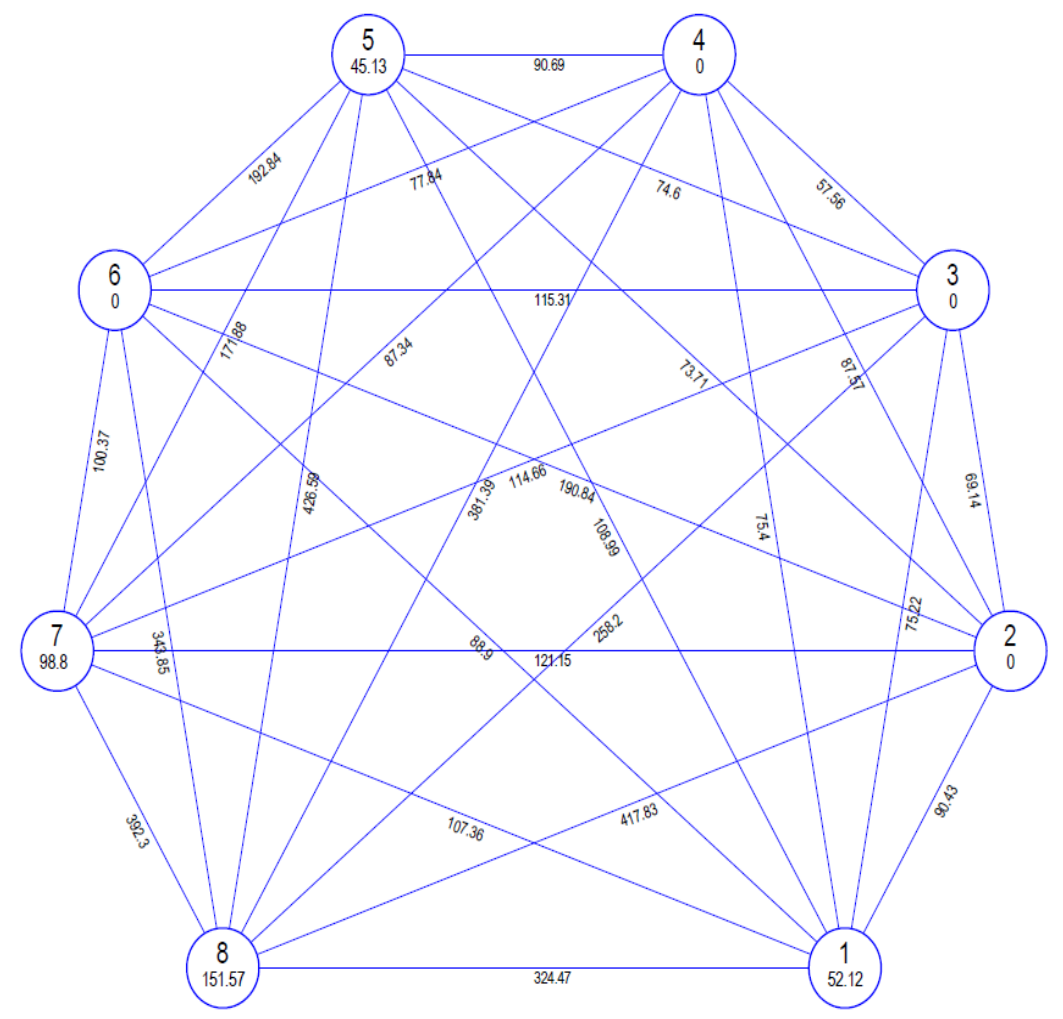

Mahalnobis Euclidean Disatnce (Not to the Scale)

Whereas, cluster IV and VI showed high mean value for single character which were number of primary branches per plant (7.17) and fruit length (26.40), respectively in $Y_{1}$ Cluster VIII showed high mean value for maximum five characters viz., node number to anthesis of first staminate flower (11.62), node number to anthesis of first pistillate flower (14.29), days to anthesis of first staminate flower (40.37), days to anthesis of first pistillate flower (40.19) and node number of first fruit harvest (14.67), cluster VI showed high mean value for inter nodal length (7.60), vine length (5.93), average fruit weight (170.50) and average fruits yield per plant (4.12) whereas, cluster II, IV and VII showed high mean value for single character which were number of primary branches per plant (8.13), days to first fruit harvest(54.63), and number of fruits per plant (28.97), respectively in $\mathrm{Y}_{2}$.
Highest per cent contribution towards total genetic divergence (Table 5) was exhibited by vine length (22.99 and 18.66) while minimum contribution was reflected by days to first fruit harvest $(0.21$ and $0.14 \%)$ in both the years, respectively. Rest of the characters exhibited low contribution towards total genetic divergence (Figs. 1 and 2). Therefore, necessary attention is required to be focused on these characters.

Intra and inter-cluster distance diagram for different quantitative characters in sponge gourd is given in Figure 3 and 6. Clustering by Tocher method shows that the lines/ $\mathrm{F}_{1}$ 's fall in to same cluster having lowest degree of divergence from each other (Figs. 4 and 5) and crosses among the lines $/ \mathrm{F}_{1}$ 's of the same cluster will be unable to produce any transgressive segregants. While, the lines $/ \mathrm{F}_{1}$ 's belonging to different clusters having 
maximum divergence can be successfully utilize in hybridization programmes to get desirable heterotic $\mathrm{F}_{1}$ 's /transgressive segregants.

\section{References}

Badade, D.S., Warade, S.D. and Gaikwad, S.K. 2001. Genetic divergence in bottle gourd. J. Maharashtra Agril. Universities, 26(2): 137-139.

Islam, M.R., Hossain, M.S., Bhuijan, M.S.R., Hasan, G.N. and Syed, A. 2010. Multivariate analysis of bitter gourd. Middle East J. Sci. Res., 5(2): 86-90.

Kalloo, G. 1993. Loofah-Luff S.P.P. (ed.). Genetic Improvement of Vegetable Crops, pp. 265-266.

Karuppaiah, P., Kavita, R. and Kumar, P.S. 2005. Correlation and path analysis in ridge gourd (Luffa acutangula L.). Crop Res., 29: 490-494.

Kumar, D., Malik, B.P.S. and Singh, V.P. 1994. Assessing genetic divergence and identification of promising parents for hybridization in field pea (Pisum sativum L.). Legume Res., 17(3-4): 225228.

Kumar, H., Srivastava, A., Vishwakarma, M.K. and Lal, J.P. 2012. Genetic enhancement of variability through induced mutagenesis in two genotypes of Brassica napus L. Madras Agric. J., 99(4-6): 228-231.
Mahalanobis, P.C. 1936. On generalized distance in statistics. Proceedings of national institute of science. 2: 49-55.

Mathew, A., Markose, B.L., Rajan, S. and Devi, S.N. 2001. Genetic divergence in bottle gourd [Lagenaria siceraria (Mol.) Standl.]. Veg. Sci., 28(2): 121123.

Panse, V.G. and Shukhatme, P.V. 1967. Statistical Methods for Agricultural Workers, $2^{\text {nd }}$ eds. Indian Council of Agriculture Research, New Delhi, pp. 235-247.

Rao, C.R. 1952. Advanced statistical method in biometrical Res., J. Wiley and Sons, Inc. New York., 15(10): 130-134.

Singh, R.K. and Chaudhary, B.D. 1985. Biometrical methods in quantitative genetic analysis, Kalyani publishers, New Delhi-Ludhiana, India. p. 318.

Sundaram, V. and Vadivel, E. 2007. Genetic divergence in bitter gourd under salt stress. Crop Res., 1/3: 139-142.

Varalakshmi, B., Reddy, Y.N. and Reddy, B.M. 1994. Genetic divergence in ridge gourd. J. Genet. Pl. Breed., 48(2): 131134.

Whitaker, T.W. and Davis, G.N. 1962. Cucurbits. Interscience Publ. Inc. New York, pp-250.

Yawalkar, K.S. 2004. Cucurbitaceous or vine crops. Vegetable Crops of India (V.ed.), p. $152-155$.

\section{How to cite this article:}

Yamuna Prasad Singh, V.B. Singh, Praveen Kumar Singh, Vimlesh Kumar and Ram Parsad. 2017. Genetic Diversity among Promising Lines and their F1 Progeny of Sponge Gourd [Luffa cylindrica (Roem) L.]. Int.J.Curr.Microbiol.App.Sci. 6(7): 2756-2768.

doi: https://doi.org/10.20546/ijcmas.2017.607.386 